\title{
Competencias digitales y educación
}

\section{Digital Competences and Education}

\author{
Luz Levano-Francia*
}

Universidad César Vallejo, Lima, Perú.

ORCID: https://orcid.org/0000-0002-3354-2653

\section{Sebastian Sanchez Diaz}

Universidad César Vallejo, Lima, Perú.

ORCID: https://orcid.org/0000-0002-0099-7694

\section{Patricia Guillén-Aparicio}

Universidad de San Martin de Porres, Lima, Perú ORCID: https://orcid.org/0000-0002-8143-3646

\section{Sara Tello-Cabello}

Universidad de San Martin de Porres, Lima, Perú ORCID: https://orcid.org/0000-0003-4864-1221

\section{Nancy Herrera-Paico}

Universidad Norbert Wiener, Lima, Perú ORCID: https://orcid.org/0000-0001-6596-3113

\section{Zoila Collantes-Inga}

Universidad Privada Telesup, Lima, Perú ORCID: https://orcid.org/0000-0002-8741-9263

Recibido 01-01-19 Revisado 21-01-19 Aprobado 13-05-18 En línea 21-05-19

\section{*Correspondencia}

Email:1levano@ucv.edu.pe
Citar como: 


\section{Resumen}

El presente artículo brinda un análisis de las competencias digitales en el contexto actual. La creciente expectativa de las nuevas tecnologías y sus múltiples aplicaciones han producido trascendentes repercusiones en todas las esferas de la sociedad, y que, en concreto, en el aspecto de la educación superior donde las demandas son más que significativas buscando que cubrir sus implicancias. En primer lugar, se da a conocer el panorama teórico sobre las diferentes acepciones y conceptos en relación con las competencias digitales. Dicho enfoque brinda información sobre aspectos que atañen a lo relacionado a las competencias digitales. Seguidamente, se presentan algunos datos que permiten dar nuevas perspectivas, tales como la importancia y repercusiones, así como cuáles deberían ser las competencias digitales en los docentes universitarios en función de la demanda actual en la educación superior. Finalmente se dan a conocer una variedad de expectativas con relación a las vertientes que podrían desarrollarse en función de tan relevante tema.

Palabras clave: Competencias digitales; Alfabetización digital; TICs; Inclusión.

\section{Summary}

This article provides an analysis of digital competences in the current context. The growing expectation of new technologies and their multiple applications have produced important repercussions in all spheres of society, and, specifically, in the aspect of higher education where the demands are more than significant seeking to cover their implications. In the first place, the theoretical panorama about the different meanings and concepts in relation to digital competences is made known. This approach provides information on aspects that relate to digital s. Next, below there are some data that allow to give new perspectives, such as the importance and repercussions, as well as what should be the digital competences in professors according to the current demand in higher education. Finally, a variety of expectations are presented in relation to the aspects that could be developed based on such a relevant topic.

Keywords: Digital Competences; Digital Literacy; ICTs; Inclusion.

\section{Introducción}

Es sin duda que el irrefrenable avance de las tecnologías del campo digital ha transformado muchos de los campos del quehacer humano. El empleo de la internet ha revolucionado los conceptos de interactividad; el empoderamiento de la información ha conducido inevitablemente a cambios en la perspectiva de la sociedad que más allá de ser significativos, han tenido y seguirán generando gran impacto y con ello cambios de tendencias cada vez más aceleradas.

Según lo manifestado por diversos investigadores acerca de los cambios tecnológicos, la sociedad en su conjunto asiste a la revolución en los modos y formas del empleo de los denominados recursos digitales y la virtualización de la información, cuyos impactos en el contexto actual busca que replantear lo que hasta ahora se tenía concebido acerca de las posibilidades de las nuevas tecnologías y sus posibles consecuencias. (Álvarez, Núñez \& Rodríguez, 2017; Mezarina, Páez, Terán \& Toscano, 2015)

La integración de las tecnologías emergentes dentro de los ambientes educativos y de la vida cotidiana ha permitido el desarrollo de los medios digitales y de los ambientes de aprendizaje electrónicos en donde los Recursos Educativos Abiertos y los Objetos de Aprendizaje expresan sus mejores potencialidades educativas (Mezarina et. al., 2015, p. 90). 
La complejidad de la sociedad actual inmersa según Galindo, Ruiz, y Ruiz (2017) en la cuarta revolución industrial, deberá enfrentar una serie de desafíos y diatribas que se amalgaman irreversiblemente y con ello se gesta la anuencia de cambios, donde las tecnologías y el creciente empleo de las mismas conlleva a diferenciar a los miembros de este contexto entre aquellos que poseen ciertas competencias digitales y los otros que oprobiosamente desconocen el impacto de su magnitud en el contexto actual (Galindo, Ruiz \& Ruiz, 2017); pero de una u otra manera son conscientes de su pertinencia en muchos de los aspectos de su entorno, ya que la sola aplicación de enorme potencialidad de este campo del saber afincado en la productividad tecnológica se encuentra, por un lado solucionando una serie de necesidades vía la digitalización de procesos (Sicilia et al., 2018). Si se asume otra postura, puede tomarse en cuenta la existencia de diversos niveles de desconocimiento, en una considerable porción de la población, que afronta la complejidad de este panorama y que por ende le resulta una situación no vinculante a su realidad, como un problema muy ausente (Bartolomé, Martínez \& Tellado 2014; Vera, Torres \& Martínez, 2014). Lo anterior no resultada nada alentador en el actual contexto, ya que según lo agenciado en la investigación llevada a cabo por la OCDE (2016) acerca del panorama de las competencias digitales entre los adultos se halló que casi la cuarta parte de los estudiados $(24,3 \%)$ desconocen completamente cómo trabajar con un computador debido a diversos factores que van desde la ausencia del mismo en su quehacer o por la forma de manejo a lo cual les resulta de extrema complejidad; pero esta cifra ya de por sí inquietante, queda muy lejos en dicho estudio donde se comprobó que en el seno del grupo remanente de los entrevistados (75\%) solo el 5\% de los mismos poseían ciertas habilidades para dar un adecuado uso del computador así como también del conocimiento de actuales herramientas informáticas adecuadas. Dicha investigación mencionó, a manera de adenda, que dos tercios de los investigados estaban en casi o completa nulidad para evidenciar la efectividad de los recursos informáticos.

\section{Acerca de las competencias digitales}

No cabe duda alguna que el siglo XXI es el siglo de la digitalización de la información, aspecto que engloba los cambios más radicales en los paradigmas que, al común del corriente, aún no se adaptan a tan severos cambios tanto cualitativos como cuantitativos. En correspondencia biunívoca con este enorme ecosistema de información que corresponde a los entornos digitales, es la necesidad de apertura de mecanismos que re-direccionen a tale cambios. Es dentro de este enfoque novedoso que se genera el trasunto correspondiente a los aspectos que engloban todo lo relacionado con las competencias digitales. Cabe mencionar que este aspecto abarca muchas esferas del accionar humano tanto académico de alto nivel, así como el gubernamental y social, (Lissitsa, Chachashvili \& Bokek 2017) que inclusive ha modificado las interacciones humanas y la forma de percibir la realidad inmediata. Al respecto de tal perspectiva, se tiene lo agenciado por Álvarez, Núñez y Rodríguez (2017) quienes en relación al aspecto académico mencionaron que lo relacionado al mundo de las competencias en el panorama digital bajo el marco de "impacto sobre el conjunto del tejido productivo, favorece la obsolescencia rápida de las competencias contempladas por las titulaciones" (p. 541); así mismo sobre este mismo aspecto Rangel y Peñaloza (2013) manifestaron que los "cambios que hoy atestigua la humanidad no solo se están dejando sentir en los ámbitos económico, político o social, sino también -y de manera crecienteen el ámbito educativo, especialmente en las Instituciones de Educación Superior"(p. 9).

Debido al avasallador avance de las tecnologías, la sociedad del conocimiento converge inevitablemente con los avances tecnológicos y con su continuo desarrollo, por lo que se ve obligada a adaptarse; con lo cual todo aquel partícipe que se oriente a este mundo deberá manejar los códigos cada vez más complejos pero muy necesarios, según lo explicado por Ocaña, Valenzuela y Garro (2019). Asumido este asunto desde otro ángulo, debe tenerse muy en cuenta los perfiles de los nuevos ciudadanos de este ecosistema universal de información, que cual similar a uno natural, establece y desarrolla sus nexos (algunas veces evidentes y otras no tanto) conllevando a una serie de niveles de sucesión en dicho proceso. Asumiendo este aspecto de consecución de factibilidades que nacen al seno del mismo desarrollo de las TICs, inteligencia artificial, entre otros, nos encontramos ad portas de un cambio generacional nunca antes visto, 
donde la interactividad es la esencia y combustible de su implementación en las sociedades a escala global, ya que no solo abarca parte de la emergente economía (tómese por ejemplo el tema de los ya famosos bitcoins), sino que está esculpiendo un nuevo prototipo de ciudadano, que a fuerza de su inminente e ineluctable adecuación se erige presto a este nuevo esquema de conectividad ¿Qué tanto en este momento deberá de tener en cuenta para este nuevo mundo de las competencias digitales?

A razón del cambiante y necesario entorno digital, las polémicas en relación a su necesidad de divulgación en relación a sus potencialidades puede obviarse, según lo manifestado por Galindo, Ruiz y Ruiz (2017) en los próximos años la urgencia de desarrollar las competencias digitales podrá enfrentar un dilema educativo a nivel superior, ya que dichas propuestas se orientan en función de las novedosas propuestas laborales, ya que según dichos autores, estamos frente a la extinción inevitable de empleos y profesiones que no estén enmarcados bajo este nuevo panorama, ya que sugestivamente demandan que este trasunto sea resuelto bajo mecanismos de alfabetización digital objetiva a nivel universitario manejando un esquema de actualización permanente. De igual manera Ocaña, Valenzuela y Garro (2018) nos refrendan similar panorama al esbozar acertadamente que asumen que las competencias digitales son aquellas requeridas en el contexto actual y que deben ser concebidas bajo las aplicaciones de las TICs y las plataformas de interactividad a tiempo real.

\section{¿Qué son las competencias digitales?}

Las denominadas competencias digitales son entendidas a manera de concepto que ha generado diversas líneas de investigación que a luz de los nuevos avances tecnológicos en el rubro de las TICs. Su vasta trascendencia en el ámbito de su aplicación a la Tecnología Educativa, cuyo espectro de acción abarca diversas proyecciones, tanto de aprendizajes, investigación, recreacionales y sociales, entre otros. Para Marzal y Cruz (2018) las competencias digitales, en la formación de los ciudadanos, permitirá perspectivas de empoderamiento en relación a aspectos sociales intrínsecos como la política, economía, empleabilidad; así como también aspectos de las nuevas tendencias culturales y de entretenimiento en el presente siglo.

Las competencias entendidas desde el panorama educativo, según lo manifestado por Marza y Cruz (2018) son asumidas a manera de instrumentos de gran utilidad que permite la movilización de actitudes, conocimientos y procesos; por medio de los cuales los discentes adquieren habilidades para facilitar la transferencia de conocimientos y generar innovación. Por su parte Iordache, Mariën y Baelden (2017) proponen que las competencias digitales se asuman como los resultados más prácticos y medibles de los procesos de formación con relación a la novedosa alfabetización digital.

Para Rangel y Peñalosa (2013) el significado de alfabetización digital, entendida como constructo, se ciñe escuetamente a procesos cognitivos que permiten la adquisición de ciertas capacidades para el empleo de las TICs y el manejo de la información, aunque para ser precisos asumen que dichos procesos son el producto de un entrenamiento en el manejo de recursos basados en tecnologías del mundo informático.

Durán, Gutiérrez y Prendes, (2016, citando a Ferrari, 2012) asumen un esquema algo diferente en relación a las competencias digitales al fundar que este aspecto es un derecho, cuyo espectro sobrepasa los límites clásicos de la formación técnica que, para redondear la idea, también deben de ser asumidos los parámetros de la gestión del conocimiento tales como mecanismos de gestión de la información y entre otras cosas más se perfila hacia un enfoque más humanista al afirmar que también se requiere de cualidades como colaboración, responsabilidad, ética, entre otros. A manera de resumen de la investigación realizada los autores señalados líneas arriba, muestran el siguiente panorama con relación a las competencias digitales las que a su 
entender comprenden diversos componentes, tales como: tecnológico, comunicativo, usos de la información y alfabetización multimedia.

Según Ocaña, Valenzuela y Garro (2019) y Krumsvik (2011, citado por Durán, Gutiérrez y Prendes, 2016) las competencias digitales deben ser entendidas bajo una visión holística que abarca saberes y capacidades de carácter tecnológico las que deben ser gestadas en primer orden a nivel de la educación superior y que, además, deben tener como sustento una red de elevada complejidad en la alfabetización tecnológica peor con carácter funcional. Dicho aspecto ha sido ya puntualizado por Rangel y Peñalosa (2013) quienes ya afirman denodadamente que para el contexto actual se requiere de nuevas competencias, habilidades y actitudes y la forma que guarda consecuencia con tal planteamiento se encuentra ligada a la implementación de un novedoso proceso de alfabetización digital.

\section{Importancia de las competencias digitales.}

¿Son realmente importantes las competencias digitales? Sobre este punto Álvarez, Núñez y Rodríguez (2017) se refirieron a la complejidad del asunto asumiendo que para las formas de interacción en el mundo de la globalización y el tránsito inevitable a la aplicación de las nuevas tecnologías en el contexto actual de la información, urge... "la necesidad de formar en nuevas competencias adaptadas al impacto de la innovación tecnológica sobre la actividad económica, impacto que se manifiesta no sólo en el ámbito profesional sino también en un sentido genérico" (p. 559). Sobre este aspecto Aguirre et al. (2015) mencionaron que el campo de las competencias es el desarrollo de habilidades relacionadas y "requeridas tanto en docentes como en estudiantes, pueden potenciar el uso de estrategias innovadoras de enseñanza - aprendizaje mediadas por la Tecnologías de información y Comunicación TIC,” (p. 90).

Acerca del desarrollo histórico del proceso relacionado con las competencias digitales los investigadores Álvarez, Núñez y Rodríguez (2017) nos mencionaron como dato que anterior al año 2008 se habían estado impulsando programas que se orientaban a la globalización de la información que según dichos autores se este aspecto está relacionado con ciertas estrategias de la Unión Europea en su Agenda Digital de Estrategias de Crecimiento para Europa 2020; ya que según las percepciones de aquel entorno vislumbraban ya importantes cambios de carácter cualitativo en relación al universo de las TICs, cambios que para la actualidad estuvieron sustentados estructuralmente ya que los cambios acaecidos y que sucederán apuntan mucho más sobre la enorme repercusión de las nuevas tecnologías. Por ejemplo, en España se ha venido desarrollando una campaña denominada $e$-skills, según lo expuesto por los autores anteriormente mencionados, que buscaba captar la atención pública sobre el mundo digital con la finalidad de ampliar el horizonte de afinidad por las novedosas tecnologías y la seguridad en la internet con lo cual lograrían ciertos cambios económico-sociales, porque se buscaba la reincorporación al mundo laboral bajo el parámetro de las TICs de cierta masa crítica en condición de desempleo.

El afianzamiento de las competencias digitales en la enseñanza superior, según lo manifestado por Mezarina et. al (2015), debe estar sujeto a la observancia de la sociedad con la finalidad de asegurar el nivel de calificación de las cualidades y competencias digitales que demanda la sociedad en un entorno y tiempo dado. Sicilia et. al (2018) asumen una postura en torno a las habilidades digitales al asumirlas como facilitadores esenciales cuyo rango de acción se requiere en labores que precisen del desarrollo e implementación de herramientas digitales según los requerimientos del entorno en el cual con solícitos. Además, hay que mencionar que no hay una uniformidad en relación a la implementación de las competencias digitales, ni siquiera en la comunidad europea, ya que el trabajo realizado por Álvarez, Núñez y Rodríguez (2017) discute y pone en evidencia que hay una distancia asincrónica en relación a tal aspecto en relación a universitarios hallando que existe una patente carencia de habilidades digitales que les ha restringido la interactividad a nivel digital, por lo que han perdido oportunidades de desarrollo; así como también una sensible debilidad en el uso y aprovechamiento de los recursos digitales y tecnologías similares que les hubiese permitido gestar nuevos conocimientos más acordes con el momento y con ello restringir la creatividad e innovación lo que traducido en el contexto actual 
trascendería como que al no desarrollar competencias digitales ha repercutido en su nivel de impacto en la economía digital y por ende en su nivel de empleabilidad, por lo que "los jóvenes no explotan al máximo las posibilidades que les brindan las nuevas tecnologías". (Álvarez, Núñez \& Rodríguez, 2017, p. 554).

La investigación realizada por Álvarez, Núñez y Rodríguez (2017) acerca de la trascendencia de las competencias digitales en estudiantes universitarios tanto de México como España, no se encuentra a la par de las exigencias laborales evidenciándose una notoria falencia en el aspecto formativo profesional en relación a dichas competencias ya que "la tendencia de un déficit en materia de alfabetización digital con lo cual puede limitar las oportunidades de empleo del alumnado" (p. 554), agenciando mucha deficiencia que según los investigadores mencionados abarcaría la totalidad de su formación universitaria; aspecto que repercutiría en su nivel de inserción laboral que sea acorde al contexto actual, con lo cual se estaría desaprovechando muchas oportunidades en relación a su impacto económico.

Sobre el trabajo realizado por Galindo, Ruiz y Ruiz (2017) con 1888 encuestados digitalmente determinaron que entre los denominados nativos digitales existe una evidente carencia de preparación para estar inmersos competitivamente en el mundo del trabajo digital y además de ello hay que tomar en cuenta que más de la mitad de ellos pasa más de tres horas conectados a la red sea por pc, computador portátil o teléfono móvil inteligente o smartphones. Lo anterior resulta de preocupación para dichos investigadores ya que, a pesar de contar con un grupo afín al mundo de las tecnologías de la información, no son capaces de extraer un mayor aprovechamiento de dichas plataformas ya que según su conclusión es la sensación de cierta autocomplacencia en el empleo de limitadas herramientas y estrategias digitales, por lo que se percibe cierta obsolescencia de competitividad digital entre los mismos, porque es una generación muy situada hacia la balanza de la vacuidad digital que se sustenta en redes sociales, interactividad de satisfacción personal; actividades que se alejan onerosamente de la generación de mejores técnicas de aprovechamiento del mundo digital. Dichas conclusiones, como se percibe, destierran lumínicamente el estereotipo tradicional que encumbraba a los nativos digitales como entes empoderados del mundo digital.

Ahora bien, adoptando una perspectiva diferente, se puede citar lo referenciado por Gil y Roca-Piera (2015) quienes pusieron de manifiesto que, según la tendencia para el año 2020 en lo que respecta a Europa, se gesta una tendencia creciente en relación a la demanda laboral en el nivel de calificación que entre otras cosas un 35\% tendrá que cubrir las exigencias de adaptación e innovación.

\section{Competencias digitales en los docentes.}

La perspectiva para asumir las posturas más trascendentales acerca de las competencias digitales que deben de manejar los maestros, se relaciona estrechamente con el sustento de que dichas competencias son inherentes a su formación y cualificación profesional, los que deben ser ajustados según sea el nivel de enseñanza en el cual se encuentren. (Álvarez, Núñez \& Rodríguez, 2017; Durán, Gutiérrez \& Prendes, 2016).

Según lo expuesto por Durán, Gutiérrez y Prendes (2016) con relación a la concepción de un docente competitivo en relación a las TICs deberá ser asumido en una posición de mayor envergadura para lo cual deberá superarse la escueta concepción que lo circunscribe al desarrollo de competencias de índole técnica o en relación a una especialidad desarrollada por el mismo.

Según la información compilada por Rangel y Peñalosa (2013) en su investigación acerca de las competencias digitales en los docentes universitarios, nos mencionan que no hay un claro conceso al respecto por lo asumen los siguientes parámetros: (a) Procesos mediados por el empleo de las TICs en donde el docente universitario deberá desarrollar ciertas destrezas en técnicas informáticas, actualización profesional constante, metodología docente acorde a los desafíos y 
actitud hacia las nuevas tecnologías; (b) Dimensiones de rasgos formativos que abarquen aspectos tales como el manejo instrumental, capacidad cognitiva, el factor actitudinal hacia las nuevas tecnologías y el afianzamiento axiológico acorde con todo lo anterior; (c) Un acercamiento hacia los mecanismos relacionados con la gestión del conocimiento que se enfoquen en aspectos esenciales tales como el manejo de nociones básicas sobre las TICs, acrecentar el nivel de conocimiento sobre las nuevas tecnologías y por último la implementación de mecanismos de generación del conocimiento. Según los autores mencionados con antelación el mundo de las competencias digitales no puede desligarse de parámetros tales como la alfabetización digital ya que, para dichos investigadores, los docentes universitarios deben desarrollar una serie de competencias que les permitan el manejo adecuado de los recursos tecnológicos, pedagógicos, informacionales, comunicativos y axiológicos.

De acuerdo a lo investigado por Batalla, Rimbau y Serradell (2014, citado por Sánchez, Sánchez \& Ramírez, 2016), los docentes universitarios de las áreas de las ciencias económicas en Extremadura, España que han asumido el reto de emplear tecnologías informáticas en su quehacer académico no han podido generar el valor agregado como herramienta generadora de conocimientos en su campo de acción debido al desconocimiento de competencias digitales adecuadas que les hubiesen permitido cierta aproximación a tales resultados. Ahora bien, respecto del asunto anterior, Fernández y Fernández (2016) mencionaron que ya desde el 2008 la Unesco había establecido tres enfoques en lo que respecta a las competencias digitales las cuales se resumen como: (1) comprensión e integración de competencias tecnológicas, (2) aplicación de los conocimientos tecnológicos a la resolución de problemas reales y concretos y, (3) producción de nuevos conocimientos a partir de los ya generados. Puede decirse al respecto que el asunto no es novedoso; pero aún hay una larga brecha por salvar en muchos países en lo que respecta a la formación de docentes competitivos en el enfoque de las competencias digitales ya que "Es evidente que un profesor no puede hacer que un alumno desarrolle una competencia que él mismo no posee en profundidad" (Fernández \& Fernández, 2016, p. 105).

\section{Perspectivas de las competencias digitales.}

El efecto del empleo de las nuevas tecnologías ha generado nuevos mecanismos de interactividad en la sociedad, los que por defecto se encuentran transformando el papel y funcionalidad de las instituciones universitarias, cuya relativa inercia era característica. No cabe duda alguna que dicho efecto ha traído y seguirá generando más y mecanismos que revolucionarán los conceptos clásicos de enfoque de interacción en el orbe.

En función de las perspectivas que continuamente se presentan en el campo tecnológico en relación al sector de la formación superior, García y Martín (2016) mencionaron que actualmente existe un consenso cuya extensión abarca vastos sectores en la sociedad por la cual se concibe el que los docentes deben ya de poseer una serie de competencias digitales necesarias con la finalidad de explotar la mayor cantidad de capacidades pedagógicas en relación a las nuevas tecnologías orientadas a la formación profesional, estructuración de novedosos planteamientos curriculares y novísimas tendencias en relación al intrincado campo de la evaluación de los aprendizajes bajo esta vertiente. Sin embargo, nos mencionan Rangel y Peñaloza (2013) para alcanzar cierto grado de materialización deseado se requiere de cierta urgencia de confirmación de aptitudes digitales que le facilitarán al docente el empleo óptimo de las TICs con lo cual efectivizarán y alcanzarán una mejor performance en relación con el desarrollo de las competencias digitales.

Según Aguirre et. al (2015) en su enfoque como habilidad acerca de las competencias digitales en el campo de la formación universitaria "se debe desarrollar a través de estrategias que presenten un grado de innovación en el proceso educativo" (p. 89). Según Gil y Roca-Piera (2015) para que los estudiantes tengan a su disposición el empleo efectivo de los recursos TICs deberán de cumplir con desarrollar ciertas competencias digitales que coadyuven a obtener el máximo o 
adecuado aprovechamiento bajo el emergente panorama del paradigma de la cultura digital. (Freire y Brunet, 2016).

Marzal y Cruz (2018) apuestan por una propuesta de un modelo educativo por competencias para la formación superior, bajo el cual se deben estructurar una serie de cambios adoptando nuevos esquemas didácticos tales como: (1) predisposición para desarrollar actitudes que viabilicen canalizar nuevas destrezas; pero de forma eficaz y eficiente al poseer técnicas y herramientas acordes con la especialidad a desarrollar; (2) desarrollo de competencias digitales inherentes a nuevas plataformas de interactividad y donde el empoderamiento del discente sea algo tangible en las múltiples formas de interactividad que se desarrollan en el seno de la digitalización de los procesos de aprendizaje. Ahora, si el tema es visto desde el panorama social Sabina, Svetlana y Ya'arit, (2017) la competencia digital deberá ser asumida como parte del derecho del individuo, que en su esencia sea más que una mera apreciación técnica ya que su campo de acción y desarrollo en el trasunto social va más allá del campo de la gestión tecnológica y administración de tecnologías desde el panorama académico.

En función de la asimilación y logro de competencias digitales, cabría preguntarse si ¿la adquisición tardía de competencias digitales sería un factor que juegue en contra de los profesionales? A primera vista pareciera que fuese contundente una respuesta afirmativa, pero claro sería desde una perspectiva obcecada al negar la versatilidad de la adquisición de capacidades en el campo de los entornos digitales, ya que según Bokek-Cohen $(2018$, p. 18) "La adquisición de habilidades digitales y la alfabetización profesional les permite a los empleados que llegan tarde en la carrera beneficiarse plenamente de sus propias fortalezas y evitar algunas de sus desventajas". Si el individuo adquiere habilidades digitales al final de su carrera, sea de manera formal o informal, puede revertir el estereotipo negativo y servir como un referente de consideración para los empleadores con respecto a la productividad potencial de sus empleados, aspecto que luego podrá derivarse en recompensas extrínsecas. (Davies \& Eynon, 2018; Lissitsa, Chachashvili \& Bokek, 2017). Y a forma de colofón sobre esta novedosa perspectiva BokekCohen (2018, p. 21) sustentó que "que adquirir y dominar las habilidades digitales es una garantía importante que demuestra la capacidad de adaptación de los trabajadores de mayor edad, su idoneidad para sus trabajos actuales y, por lo tanto, su mérito para obtener mayores recompensas extrínsecas".

\section{Conclusiones}

Según los estudios realizados por la OCDE (2016) acerca del panorama actual en relación a las capacidades y habilidades interpersonales, el nivel de competencia en relación al procesamiento de la información y otra gama de habilidades de carácter cognitivo de alto nivel, son cada vez más que necesarias ya que su rango de acción va más allá del aspecto profesional, sino que en el campo empresarial en diversos estamentos de alta vertiente comercial tales como la gestión y administración digital, comercio digital y el mundo del manejo de la bastedad de datos que se generan en cada segundo, rubro en el cual la inteligencia artificial es la llamada a refundar la concepción de las potencialidades de la nuevas tecnologías. (Ocaña, Valenzuela \& Garro, 2019)

Conforme se desarrollen nuevos avances y con ello se reorienten o generen nuevos perfiles en el seno universitario frente a las demandas de la sociedad en su conjunto (Morán, Cardoso Cerecedo Ortíz, 2015), demandarán de las instituciones universitarias una reflexión sobre su oferta académica, la cual por lo que respecta al contexto que se está desarrollando, dicha oferta en su planeamiento tendrá que refrendarse una variedad de competencias de tipo transversal que guarden concordancia con los perfiles exigidos en relación las TICs. (Ocaña, Valenzuela y Garro, 2019; Gil y Roca-Piera, 2015). Al respecto Porlán, Espinosa y Sánchez (2018), mencionaron sobre la contundencia de las florecientes tecnologías emergentes sustentada en inteligencia artificial (IA) sobre el quehacer educativo sigue en un auge creciente de interés a nivel global ya que brinda los mecanismos más accesitarios para las demandas del sector, en especial en aquellos países con economías emergentes, que es donde su impacto está generando grandes cambios. Para que el proceso sea de carácter inclusivo, hay que tener en cuenta lo 
expuesto por Davies y Eynon (2018) que plantearon que todas aquellas propuestas o discursos orientados a sustentar el cómo deberá de estructurarse y practicarse el enfoque sobre el desarrollo de programas de competencias digitales, deberá estar sustentado en las necesidades y el mercado de las nuevas generaciones de forma tal que todo ello sea un aporte generacional.

Para finalizar en relación con la reverencia y relevancia de las competencias digitales en función de la sociedad y en especial de las universidades, lo manifestado por (Freire y Brunet, 2016, p. 86) que lo rubricaron claramente al manifestar que "Nuestras escuelas y universidades no pueden permanecer ya al margen de esta transformación". La universidad urge de sendas transformaciones de carácter académico, organizacional, humanístico y científico, sino no podrá enfrentar las nuevas perspectivas del floreciente panorama digital. La manera en que se encare la nueva educación digital traerá consecuencias para aquella sociedad en la que es subestimada todo lo relacionado con el desarrollo y aplicación de nuevas tecnologías. Más allá de los promisorios discursos de prosperidad, que enfatizan el imperativo de maximizar la riqueza individual, se requiere con prístina celeridad hallar las formas de generar el fomento, valoración y recompensas orientadas al logro de las competencias digitales que puedan conducir a una sociedad más inclusiva y socialmente cohesionada.

\section{Referencias}

Álvarez, E., Núñez, P., \& Rodríguez, C. (2017). Adquisición y carencia académica de competencias tecnológicas ante una economía digital. Revista Latina de Comunicación Social, 72, 540-559. Doi: http://dx.doi.org/10.4185/RLCS-2017-1178

Bartolomé, A., Martínez, E., \& Tellado, F. (2014). La evaluación del aprendizaje en red mediante blogs y rúbricas: ¿complementos o suplementos? REDU: Revista de Docencia Universitaria, 12(1), 159. Doi: https://doi.org/10.4995/redu.2014.6430

Bokek-Cohen, Y. (2018). Conceptualizing employees' digital skills as signals delivered to employers. International Journal of Organization Theory \& Behavior, 21(1), 17-27. Doi: https://doi.org/10.1108/ijotb-03-2018-003

Centeno Moreno, G., \& Cubo Delgado, S. (2013). Evaluación de la competencia digital y las actitudes hacia las TIC del alumnado universitario. Revista de Investigación Educativa, 31(2), 517-536. Doi: http://dx.doi.org/10.6018/rie.31.2.169271

Cervera, M. G., Martínez, J. G., \& Mon, F. M. E. (2016). Competencia digital y competencia digital docente: una panorámica sobre el estado de la cuestión. Revista Interuniversitaria de Investigación en Tecnología Educativa, 0, 74-83. Doi: http://dx.doi.org/10.6018/riite/2016/257631

Davies, H. C., \& Eynon, R. (2018). Is digital upskilling the next generation our "pipeline to prosperity"? New Media \& Society, 20(11). Doi: http://dx.doi.org/10.1177/1461444818783102

Durán, M., Gutiérrez, I., \& Prendes, M. (201). Análisis conceptual de modelos de competencia digital del profesorado universitario. RELATEC: Revista Latinoamericana de Tecnología Educativa, 15(1), 97-114. Doi: https://doi.org/10.17398/1695-288X.15.1.97

Esteve Mon, F. M. (2015). La competencia digital docente: análisis de la autopercepción y evaluación del desempeño de los estudiantes universitarios de educación por medio de un entorno 3D. Recuperado de: http://hdl.handle.net/10803/291441

Fernández-Cruz, F. J., \& Fernández-Díaz, M. J. (2016). Los docentes de la Generación Z y sus competencias digitales. Comunicar, 24(46), 97-105. Doi: http://dx.doi.org/10.3916/C462016-10

Freire, J., \& Brunet, K. S. (2016). Políticas y prácticas para la construcción de una Universidad Digital. La cuestión universitaria, 6, 85-94. Recuperado de: http://polired.upm.es/index.php/lacuestionuniversitaria/article/view/3388

Galindo, F., Ruiz, S., \& Ruiz, F. (2017). Competencias digitales ante la irrupción de la Cuarta Revolución Industrial. Estudos em Comunicação, 25(1), 1-11. Recuperado de: http://ojs.labcom-ifp.ubi.pt/index.php/ec/article/view/277 
García, A., \& Martín, M. (2016). Análisis de las competencias digitales de los graduados en titulaciones de maestro, 15(2), 155-168. Doi: https://doi.org/10.17398/1695288X.15.2.155

Gil Serra, A., \& Roca-Piera, J. (2015). Movilidad virtual, reto del aprendizaje de la educación superior en la Europa 2020. Revista De Educación a Distancia, 26, 1-16. Recuperado de: https://revistas.um.es/red/article/view/231941

Iordache, C., Mariën, I., \& Baelden, D. (2017). Developing Digital Skills and Competences: A QuickScan Analysis of 13 Digital Literacy Models. Italian Journal of Sociology of Education, 9(1), 6-30. doi: https://doi.org/10.14658/pupj-ijse-2017-1-2

Lissitsa, S., Chachashvili, S., \& Bokek, Y. (2017). Digital skills and extrinsic rewards in late career. Technology in Society, 51, 46-55 p., Doi: https://doi.org/10.1016/j.techsoc.2017.07.006

Marza, M., \& Cruz, E. (2018). Gaming como Instrumento Educativo para una Educación en competencias Digitales desde los Academic Skills Centres. Revista General de Información y Documentación, 28(2), 489-506. Doi: http://dx.doi.org/10.5209/RGID.60805

Mezarina, C., Páez, H., Terán, O., \& Toscano, R. (2015). Aplicación de las TIC en la educación superior como estrategia innovadora para el desarrollo de competencias digitales. Campus Virtuales, 3(1), 88-101. Recuperado en: http://uajournals.com/ojs/index.php/campusvirtuales/article/view/52

Morán, R.; Cardoso, E.; Cerecedo, M. y Ortíz, J. (2015). Evaluación de las Competencias Docentes de Profesores Formados en Instituciones de Educación Superior: El Caso de la Asignatura de Tecnología en la Enseñanza Secundaria. Formación Universitaria, 8(3), 57-64. Doi: http://dx.doi.org/10.4067/S0718-50062015000300007

Porlán, I. G., Espinosa, M. P. P., \& Sánchez, F. M. (2018). Competencia digital: una necesidad del profesorado universitario en el siglo XXI. RED: Revista de Educación a Distancia, 56, 7. Recuperado de: https://dialnet.unirioja.es/servlet/articulo?codigo=6501069

Ocaña-Fernández, Y., Valenzuela-Fernández, L., y Garro-Aburto, L. (2019). Inteligencia artificial y sus implicaciones en la educación superior. Propósitos y Representaciones, 7(2), 17 pp. Doi: http://dx.doi.org/10.20511/pyr2019.v7n2.274

OECD. (2016). Skills Studies. Recuperado de: https://www.oecd.org/centrodemexico/laocde/

Rangel, A., \& Peñalosa, E. (2013). Alfabetización digital en docentes de educación: construcción y prueba empírica de instrumento de evaluación. Píxel-Bit. Revista de Medios y Educación, 43, 9-23. Doi: http://dx.doi.org/10.12795/pixelbit.2013.i43.01

Ruiz, M., \& Belén, A. (2016). El profesorado universitario y las TIC. Análisis de su competencia digital. Ensayos: Revista de la Facultad de Educación de Albacete, 31(1), 133-147. Doi: https://doi.org/10.18239/ensayos.v31i1.1033

Sánchez, M. R. F., Sánchez, M. S. O., \& Ramírez, R. R. (2016). La evaluación de la competencia digital en la docencia universitaria: el caso de los grados de empresariales y económicas. Revista Colombiana de Ciencias Sociales, 7(2), 332-348. Recuperado de: http://www.funlam.edu.co/revistas/index.php/RCCS/article/view/1726

Sicilia, E. García-Barriocanal, S. Sánchez-Alonso, P. Różewski, M. Kieruzel, T. Lipczyński, C. Royo, F. Uras, \& S. Hamill. (2018). Digital skills training in Higher Education: insights about the perceptions of different stakeholders. In Proceedings of the 6th International Conference on Technological Ecosystems for Enhancing Multiculturality (TEEM 2018) (Salamanca, Spain, October 24-26, 2018), F. J. García-Peñalvo Ed. ACM, New York, NY, USA, 7 pages. https://doi.org/10.1145/3284179.3284312

Vera Noriega, J. Á., Torres Moran, L. E., \& Martínez García, E. E. (2014). Evaluación de competencias básicas en TIC en docentes de educación superior en México. Píxel-Bit. Revista de Medios y Educación, (44), 143-155. Doi: https://doi.org/10.12795/pixelbit.2014.i44.10 